\title{
El círculo de conversación como estrategia didáctica: Una experiencia para reflexionar y aplicar en educación superior
}

\author{
Conversation Circles as a Teaching Strategy: \\ An Experience to Reflect on and Implement in Higher Education
}

\author{
Mónica Arias Monge \\ Departamento de Docencia Universitaria \\ Universidad de Costa Rica \\ San José, Costa Rica \\ monicaariasm@hotmail.com
}

Recibido 17 de abril de 2012 • Corregido 12 de junio de 2012 • Aceptado 28 de junio de 2012

\begin{abstract}
Resumen. La experiencia que presento a continuación tiene como propósito la reflexión, fundamentación, planificación y aplicación del círculo de conversación como estrategia didáctica, en la asignatura "PF-4237 Teoría de la Educación: Multiculturalidad y Educación", del Programa de Doctorado Latinoamericano en Educación, de la Universidad de Costa Rica. Experiencia de formación que tiene como fundamento la teoría de la acción comunicativa, orientada a integrar el acompañamiento docente, la confrontación a la alteridad y la construcción de conocimientos, habilidades y actitudes sociales en educación superior.
\end{abstract}

Palabras claves. Estrategia didáctica, círculo de conversación, experiencia, educación superior.

Abstract. The experience approached in this paper aims at reflecting, reasoning, planning and implementing the "Conversation Circles" as a teaching strategy in the PF-4237 course "Theory of Education: Multiculturalism and Education" of the Latin American Doctoral Program in Education, University of Costa Rica. This training experience, based on the communicative action theory, intended to integrate the assistance of the teacher, the confrontation to otherness and the building of knowledge, skills and social attitudes in higher education

Keywords. Teaching strategy, conversation circle, experience, higher education.

\footnotetext{
Doctora en diversidad y cambio en Educación de la Universidad de Barcelona. Máster en formación y desarrollo de recursos humanos de la Universidad Internacional de Andalucía. Docente del Doctorado Latinoamericano en Educación y del Departamento de Docencia Universitaria de la Universidad de Costa Rica.
} 


\section{Introducción}

La invitación a participar como docente del Programa de Doctorado Latinoamericano en Educación de la Universidad de Costa Rica, en el segundo semestre de 2011, específicamente en la asignatura "PF-4237 Teoría de la Educación: Multiculturalidad y Educación", constituyó un nuevo desafío en el trayecto de la reflexión y acción pedagógica en la docencia universitaria.

En primer lugar, representó un desafío, porque como docente entiendo que la acción pedagógica está mediada por unas prescripciones institucionales y unas relaciones humanas en las que se suscitan unas determinadas formas de pensar, hacer, estar y ser en la universidad. Las que me convocan en cada curso lectivo, en cada asignatura, en cada clase planificada, desarrollada y evaluada, a cuestionarme, continuamente, sobre: ¿a quién?, ¿cuándo?, ¿por qué?, ¿cómo? y ¿para qué enseño? Preguntas sobre la acción pedagógica que me remiten a la consideración de la "mayéutica" como principio del acto de formación, en el que puede asumirse el vértigo de la comprensión del "sí mismo" y dar a luz el pensamiento del "otro". Que me llevan a pensar en cuestiones fundamentales como lo son el poder y el conocimiento; en esa relación que establezco como docente con el estudiantado, y que ellos y ellas establecen conmigo; y en cómo gestar, en el acto formativo, en la planificación, en el desarrollo y en la evaluación didáctica del curso, la confrontación a la alteridad en la construcción de conocimientos, habilidades y actitudes sociales.

En segundo lugar, planteó un desafío, porque el tema y el objetivo del programa de la asignatura proponían el abordaje de las problemáticas y teorías de la educación, referentes a la inclusión, la diversidad, la diferencia, la singularidad, la subjetividad y las relaciones de alteridad; así como las implicaciones políticas, prácticas e investigativas de la relación entre educación y cultura. Además, el programa de la asignatura establecía que los estudiantes debían conocer, analizar, comprender y reconocer problemáticas y teorías sobre los temas mencionados.

Estos temas y objetivos me llevaron, como docente, a reflexionar en cómo ponerme y exponerme en la relación pedagógica, en una cultura más o menos compartida, pero en la que habría un camino por dibujar, por hacer y luego por reconocer. Y en donde tendría el reto de acompañar al estudiantado a leer, escuchar, observar, sentir, vivir y escribir y, por tanto, a querer estar en relación con las palabras, los sentimientos, los pensamientos y las vidas de los otros. Es decir, a esa relación de humanidad en la que se construye, con el lenguaje, lo que se piensa y lo que se hace como una experiencia educativa.

Y, en último lugar, ese reto se intensifica porque, según lo dispuesto por el Programa de Doctorado Latinoamericano en Educación, las asignaturas son desarrolladas por dos docentes. Es decir, la docencia se comprende como una acción compartida, lo que implicaba tener que abrir las puertas para los espacios y tiempos para el diálogo, para el consenso y para la divergencia sobre la acción pedagógica. 
Los tres desafíos a los que me confrontó el curso "PF-4237 Teoría de la Educación: Multiculturalidad y Educación" me llevaron a pensar en la selección y aplicación de una estrategia didáctica que permitiera integrar el desarrollo de la docencia compartida, el acompañamiento de una experiencia educativa y la confrontación a la alteridad en la construcción de conocimientos, habilidades y actitudes sociales de del grupo de estudiantes del doctorado.

\section{Propósito}

Los desafíos e inquietudes que me llevaron a justificar y a fundamentar una experiencia docente e investigativa se originaron en el siguiente propósito:

Aplicar el círculo de conversación como estrategia didáctica en el curso "PF-4237 Teoría de la Educación: Multiculturalidad y Educación", para integrar el desarrollo de la docencia compartida, el acompañamiento de una experiencia educativa y la confrontación a la alteridad en la construcción de conocimientos, habilidades y actitudes sociales del estudiantado del Programa de Doctorado Latinoamericano en Educación.

\section{Aproximaciones a la fundamentación metodológica de los círculos de conversación}

Comprendo la docencia como una acción compleja en la que se trasversalizan conocimientos y saberes, teorías y prácticas, métodos y experiencias, en un diálogo incesante entre docencia e investigación. Por lo que los desafíos e inquietudes que me proponía la asignatura "PF-4237 Teoría de la Educación: Multiculturalidad y Educación" se cruzaban ineludiblemente con la consideración de la acción formativa como un proceso comunicacional, en cuanto a participación y compromiso (García, 2007).

Estas consideraciones me llevaron a profundizar en los principios del dialegesthai y en los fundamentos metodológicos del enfoque socio-crítico y comunicativo-crítico; en donde emerge la conversación como estrategia de indagación, de investigación y de argumentación, y en sus posibilidades de utilización en la didáctica.

Primero, como estrategia de indagación e investigación, porque al hablarnos los unos con los otros, sin pretensiones explícitas de dirección o interpretación, emerge el mundo en su objetividad-subjetividad, suscitándose la interacción emancipadora del discurso (Gadamer, 2004).

Segundo, como estrategia de argumentación porque si consideramos "(...) la conversación y su desarrollo como un todo, la serie de ideas entrelazadas y la manera en que éstas van avanzando en constante interdependencia (...)" (Elias, 1990, p. 40), 
URL: http://www.una.ac.cr/educare

podemos advertir que se trata de un fenómeno en el que las ideas de uno u otro interlocutor pueden variar y producir consensos o divergencias y líneas de apertura de significados y sentidos.

Aunque estos argumentos dan sustento al proceso comunicacional, en mi opinión, requerían de una fundamentación teórica y metodológica en la que se pudiera planificar una acción formativa, sin que la lógica de la planificación didáctica rompiera con la riqueza emancipadora y creadora del proceso dialógico.

Así que, después de un largo trabajo de revisión documental y de formación, encontré la fundamentación que requería en los círculos de conversación, una metodología fundamentada en los círculos de paz y utilizada en la educación para la paz y en la resolución alternativa de conflictos, que propicia el desarrollo de habilidades y actitudes comunicativas (Martínez, 2010).

\section{El círculo de conversación como estrategia didáctica}

\section{Principios del círculo de conversación en la planificación didáctica}

El "Círculo" no actúa de manera mágica; más bien proporciona un foro -con un marco filosófico y una estructura- en el cual es altamente probable que ocurra un cambio profundo (Pranis et al. citada por Martínez, 2010, p. 46).

La estrategia propuesta del círculo de conversación se fundamenta en el trabajo de investigación y experiencia de Pranis, Stuart y Wedge (2003) y en el estudio de la Comisión Nacional para el Mejoramiento de la Justicia, desde donde se ha promovido, en Costa Rica, la formación y utilización de los "círculos" en diferentes contextos educativos, de grupos comunitarios, de gobiernos locales y del sector corporativo (Bernal y Castillo, 2006).

La estrategia del círculo de conversación se argumenta en la sabiduría de grupos y tradiciones indígenas de diferentes partes del mundo y en los conocimientos de las teorías dialógicas, de la resolución pacífica de conflictos, de la educación popular, de la educación holística y de la teoría de la dinámica de grupos (Martínez, 2010).

Por lo que el propósito de utilizar el circulo de conversación como estrategia didáctica está orientado a estimular la reflexión personal y colectiva, mediante actividades en las que las personas que participan puedan hablar y ser escuchadas. Y, en este sentido, es importante destacar que no pretenden conducir a las personas participantes al logro de un objetivo determinado, buscan únicamente que las personas participantes aprendan de los otros y mejoren sus relaciones sociales (Pranis et al., 2003), 
Gregory Bateson, que se dedicó largo tiempo a la necesidad de que se reconocieran las interacciones, los procesos mentales que unen a todos los sistemas vivos y la necesidad de promover procesos de integración, explica la importancia y la urgencia de una nueva epistemología, de recuperar una posición, de ser como criatura parte de la totalidad. "En la cosmología totémica de los aborígenes australianos existía un sistema que ponía en relación todas las especies, las fuerzas naturales y las instituciones humanas, las plantas y los animales, el viento y el trueno, la circuncisión y el boomerang; el sistema definía la posición del hombre en aquella compleja totalidad y le permitía utilizar el sentido de aquellas múltiples relaciones en las decisiones de la vida. El campesino del Medioevo europeo iba a arar los campos en presencia de una muchedumbre (o nube) de testimonios: santos patronos, potestades y principiantes y, naturalmente, ángeles. La verdad común al aborigen y al campesino es la verdad de la integración. Hoy, en cambio, hay motivos para preocuparnos, porque podemos ciertamente hacer aprender a nuestros hijos una larga serie de hechos concernientes al mundo, pero a continuación nos damos cuenta de que ellos no saben como ponerlos en relación en una sola visión unificada: falta la "estructura que conecte". Haber perdido una posición en la totalidad compleja es lo que impide, aún aprendiendo muchas nociones, elaborar y madurar un saber capaz de conectar en una visión unificada lo que, por otra parte, sigue siendo información sin vínculos, que no se inserta en un tejido de relaciones, que sitúa lo que hemos aprendido y a nosotros mismos que lo aprendemos. Precisamente para recuperar y reactivar procesos mentales capaces de re-situarnos como siendo-parte de una totalidad es necesario volver a abrir una economía entre cielos y tierra: reencontrar nuestro lugar y nuestras competencias. (De Vita, 2009, p. 75)

El proceso de enseñanza y aprendizaje es mediado por un acto comunicacional, en donde se privilegia la consideración de la integralidad de la vida humana, mediante la realización de actividades lúdicas y socializadoras. Por lo que se comprende al ser humano desde una visión holística en la que se reconoce las características y cualidades intelectuales, corporales, emocionales y espirituales de cada sujeto, para la potenciación del análisis y síntesis de información, de la creatividad, de la expresión de los sentimientos, de la autorreflexión, de los valores, del lenguaje y cuidado corporal, del reconocimiento de intereses, necesidades y diferencias de otros sujetos.

La utilización del círculo de conversación como estrategia didáctica posibilita diferentes formas de abordar un tema específico, mediante la significación y búsqueda de sentido, en las construcciones de conocimiento, de habilidades y de actitudes socializadas.

La planificación didáctica del círculo de conversación partió de la consideración de los siguientes principios básicos, comunes a todo círculo de conversación, independientemente de las razones que lo motivan: 
URL: http://www.una.ac.cr/educare

- La participación es voluntaria y debe ser motivada por las personas acompañantes, de acuerdo con los intereses, necesidades y diferencias de los sujetos participantes.

- Las diferencias singulares de los sujetos participantes se valoran y no son ocultadas o minimizadas; por el contrario, son reconocidas como expresión de la riqueza de la diversidad existente en todo grupo humano.

- Las personas se sientan en círculo, equidistantes del punto central, por lo que la ubicación espacial remite a una relación cara a cara, en igualdad, sin jerarquías. Consideración que rompe con las estructuras tradicionales de disposición de poder y conocimiento, desplazando el poder y el conocimiento a otros puntos y a otras personas.

- Plantea un reto a las personas participantes: poder llegar a ser, es decir, trasladar las habilidades y actitudes que se practican en el "Círculo" a la vida cotidiana, como instrumento para promover una cultura de diálogo, de reflexión y democracia.

- Invitan al trato respetuoso y a la búsqueda de consensos, con el propósito de lograr nuevas formas de trabajo y convivencia (Martínez, 2010).

Por lo tanto, la consideración de la integralidad del ser humano favorece una planificación didáctica desde una posición en la que se reconoce la autoridad del conocimiento y del saber de mujeres y hombres, que puede circular como construcción social.

\section{Elementos estructurales del círculo de conversación}

A continuación se conceptualizan los elementos estructurales del círculo de conversación considerados en la planificación didáctica:

- Valores: Las personas que participan en el círculo de conversación nombran, definen y consensúan valores que consideran importantes en su vida, y que operan como referentes normativos del comportamiento y de la reflexión en la realización de las actividades.

- $\quad$ Ceremonias de apertura y de cierre: Preparan a las personas participantes del círculo de conversación para iniciar un proceso de formación cualitativamente distinto, mediante actividades, lecturas y reflexiones que invitan a estar presente, a serenarse, a abrirse a nuevas posibilidades de interpretación, comprensión y aprendizaje, a estar concientes de lo que se dice y de lo que se hace.

- Piezas del diálogo: Objetos que otorgan el derecho de hablar y de ser escuchadas a las personas participantes del "Círculo de conversación"; circulan de mano en 
mano propiciando el aprendizaje de la escucha y del silencio respetuoso. Las rondas con las piezas del diálogo permiten profundizar sobre teorías y prácticas y llegar a consensos, conduciendo a construcciones socializadas de conocimientos, habilidades y actitudes.

- $\quad$ Pueden ser objetos escogidos por las personas acompañantes o por las personas participantes; usualmente son objetos que simbolizan algo referente a los participantes o al tema que se aborda.

- Acompañantes: Personas que cuidan y acompañan el ambiente del "Círculo de conversación", se encargan de planificar, ejecutar, sintetizar, analizar y evaluar el proceso de formación. La participación de las personas acompañantes es importante, pero no tienen un rol de control; su tarea más significativa es cuidar el ambiente a lo interno del "Círculo", para que se cuente con un ambiente grupal seguro para el diálogo. Las personas acompañantes no requieren de una formación especializada, pero si de una actitud crítica y vigilante de los intereses y necesidades de cada sujeto, una actitud de apertura y aceptación hacia las personas, el compromiso de mejorar la forma de comunicación, el compromiso de participar activamente en la construcción de una sociedad pacífica, ser reflexivos, organizados y flexibles.

- Consenso: Se llega mediante la experiencia en la que las personas participantes interpretan y comprenden los argumentos y posiciones de los otros, como visiones que no son antagonistas y como parte de una relación de intercambio y de construcción colectiva (Martínez, 2010).

\section{Etapas del círculo de conversación}

La planificación didáctica de los círculos de conversación requiere considerar las siguientes etapas:

Discernimiento: En esta etapa se determina la aplicabilidad del círculo de conversación. Es decir, se decide si la estrategia es adecuada para la población y el tema que se va a abordar. En esta etapa de investigación se recoge información sobre el tema, las características, intereses y necesidades de las personas, y se definen posibles objetivos para el desarrollo de la clase.

Preparación: Comprende la preparación e información a las personas con las cuales se realizará el círculo. Así como la preparación de los aspectos logísticos como: hora, lugar, refrigerio, actividades a desarrollar y piezas de diálogo que se van a utilizar.

Realización: La realización se desarrolla también en cuatro fases que comprenden: crear confianza, construir relaciones, trabajar el tema y profundizar en las reflexiones. 
URL: http://www.una.ac.cr/educare

En esta etapa es importante considerar que es recomendable dar el tiempo necesario a la realización de cada fase, el cual dependerá de las características y cualidades de cada participante, del tema a abordar y de la dinámica de las relaciones entre las personas. Esto implica estar abierto y ser flexible a trabajar con un tiempo que no coincide exactamente con el planificado.

Seguimiento: Esta etapa se realiza con el propósito de promover cambios y mejoras al proceso formativo y con el propósito de recoger la esencia de la experiencia que se puede compartir como saber construido socialmente (Martínez, 2010).

\section{Planificación didáctica de una clase con el círculo de conversación}

Habían transcurrido las tres primeras clases de la asignatura "PF-4237 Teoría de la Educación: Multicultura y educación" y había ido siguiendo el ritmo de las palabras en los diarios y de las voces en las clases de las personas participantes: -interculturalidad, multiculturalidad, educación inclusiva y equidad-; palabras y voces que resonaban en el espacio de la clase, casi perfectas, casi completas, casi incuestionables y casi vacías, que me remitían a lugares y tiempos fijos, a referentes teóricos concretos y a "verdades" pintadas de absoluto.

Los participantes eran hombres y mujeres, de edades comprendidas entre los 30 y 50 años, de diferentes nacionalidades, profesionales de diferentes áreas disciplinares y del conocimiento y con experiencias culturales y educativas diversas. Pero las palabras de las tres primeras sesiones en muy poco remitían a esas personas, a esos contextos, a esas relaciones y a esas experiencias.

Considerando la pertinencia de integrar el desarrollo de la docencia compartida, el logro de los objetivos propuestos en el programa en el desarrollo de una experiencia educativa, la confrontación a la alteridad en la construcción de conocimientos, habilidades y actitudes sociales del grupo de estudiantes del doctorado, las características y cualidades de las personas participantes y considerando el fundamento teórico metodológico de los círculos de conversación; propuse desarrollar la cuarta sesión de clase utilizando el círculo de conversación como estrategia didáctica.

El tema propuesto fue "Derechos humanos y poblaciones de riesgo: políticas y prácticas" y el objetivo del círculo de conversación propuesto fue compartir diferentes perspectivas teóricas y experienciales sobre los derechos humanos, la cultura y la educación. 
Tabla 1

Planificación didáctica de una clase con círculos de conversación

\begin{tabular}{|c|c|}
\hline Etapas & Descripción \\
\hline Discernimiento & $\begin{array}{l}\text { - Estudiantes del Doctorado Latinoamericano en Educación que cursan la asignatura de } \\
\text { "Teoría de la Educación: Multiculturalidad". } \\
\text { - Se planifica para el desarrollo del tema "Derechos humanos y poblaciones de riesgo: } \\
\text { políticas y prácticas" } \\
\text { - El objetivo es: compartir diferentes perspectivas teóricas y experienciales sobre los } \\
\text { derechos humanos, la cultura y la educación. }\end{array}$ \\
\hline Preparación & 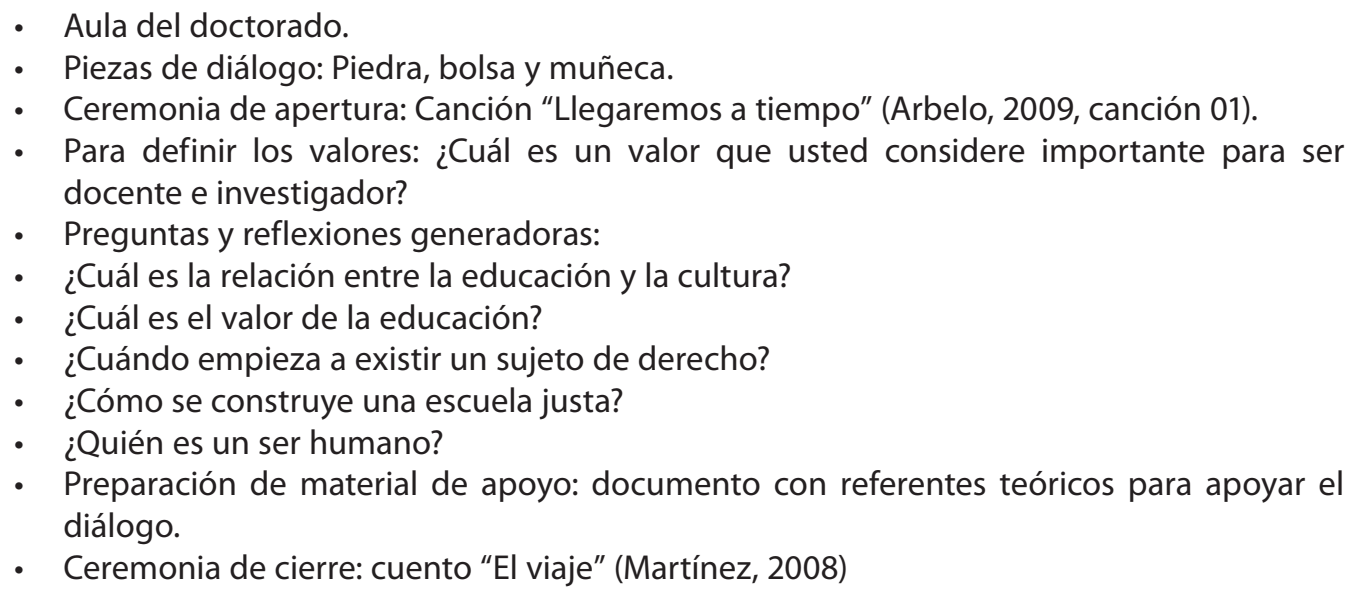 \\
\hline $\begin{array}{l}\text { Realización } \\
\text { círculo }\end{array}$ & $\begin{array}{l}\text { - Bienvenida y presentación sobre la sesión. Tiempo aproximado: } 5 \text { minutos } \\
\text { - Explicación del uso de la pieza del diálogo } \\
\text { - Explicación del círculo de conversación. Tiempo aproximado: } 10 \text { minutos. } \\
\text { - Ceremonia de apertura: canción "Llegaremos a tiempo". (Arbelo, 2009, canción 01). } \\
\text { - Tiempo aproximado: } 10 \text { minutos. } \\
\text { - } \quad \text { Trabajo en la definición de valores. Tiempo aproximado } 35 \text { minutos. } \\
\text { - } \text { apoyo de material documental de referentes teóricos. Tiempo aproximado: } 35 \text { minutos. } \\
\text { - } \quad \text { Diálogo con el tema elegido mediante preguntas definidas. Tiempo aproximado: } 1 \text { hora } \\
\text { - } \text { y } 15 \text { minutos. }\end{array}$ \\
\hline Seguimiento & $\begin{array}{l}\text { - Consulta sobre cómo se han sentido en la experiencia } \\
\text { - Se decide si se efectúa nuevamente la estrategia, si quienes participan externan el interés } \\
\text { en repetir la experiencia. }\end{array}$ \\
\hline
\end{tabular}

En la etapa de la planificación didáctica de discernimiento se consideraron los fundamentos y principios expuestos en los apartados anteriores, y las características y cualidades de las personas participantes. También se compartió, según lo dispuesto, con la docente acompañante la definición del tema y del objetivo de la sesión de clase. 
URL: http://www.una.ac.cr/educare

Para la etapa de preparación se consideraron los siguientes elementos estructurales:

- Las piezas del diálogo que se seleccionaron refieren a objetos típicos de los países representados por las personas participantes.

- La ceremonia de apertura que se propuso fue escuchar una canción que alude al valor de los sueños, a la constancia, a la voluntad y a la solidaridad en la materialización del sueño en un proyecto común. La selección de la canción se fundamentaba en la pertinencia de motivar el trayecto del doctorado como una travesía compartida y como un proyecto posible, ya que durante las tres primeras sesiones de clase había advertido que las personas participantes estaban muy angustiadas por la cantidad de lecturas, de trabajos y por aprobar el curso, pero que disfrutaban poco de la experiencia que vivían.

- La pregunta para definir los valores derivó de la necesidad de buscar referentes y experiencias comunes, que permitieran significar y dar sentido a lo que se vive, piensa y siente. Así que como todos y todas son docentes e investigadores, se partió de esa búsqueda de experiencias singulares y comunes en un contexto que nos fuera más o menos conocido.

- Las preguntas que se plantearon para desarrollar el diálogo y la reflexión son el resultado del análisis de los temas y objetivos del curso, esta parte comprendió la realización de un mapa conceptual y la selección de fragmentos de artículos y libros que pudieran orientar y fundamentar teóricamente el diálogo. También en esta etapa se preparó un material impreso, el cual contenía una breve descripción de los círculos de conversación, la planificación didáctica de la clase y los fragmentos de referentes teóricos que apoyarían el diálogo y la reflexión.

- $\quad$ Para la ceremonia de cierre se propuso la lectura compartida del cuento "El viaje", relato que refiere a historias y a la posibilidad de pensar en nuevas formas de hacer en educación, a partir de la consideración de la diferencia y de la diversidad en los contextos, relaciones y experiencias en las que los seres humanos piensan, sienten y viven.

En la etapa de planificación didáctica de la realización se consideraron las fases y tiempos de ejecución de cada actividad.

Y, finalmente, en la planificación didáctica de la etapa de seguimiento se consideró el realizar una reflexión en el diario sobre la experiencia vivida en el círculo de conversación. 


\section{Realización de una clase con el círculo de conversación}

\section{Antes de empezar}

Para describir lo que aconteció aquella mañana del martes 30 de agosto de 2011 en la sala del Programa de Doctorado Latinoamericano en Educación, tengo que empezar describiendo mis percepciones iniciales, antes de entrar al aula.

En primer lugar, era mi primer año como docente del Programa de doctorado Latinoamericano en Educación, y me preocupaba ser demasiado transgresora con mis formas de pensar y hacer.

En segundo lugar, me angustiaba desarrollar una estrategia innovadora, en un ambiente típicamente académico, en el que usualmente al docente se le llama doctor o doctora, en el que la disposición de las mesas y las sillas es fija, y en el que el docente desarrolla clases magistrales. Me angustiaba porque el círculo de conversación implicaba romper con esas normas académicas en las que se comprenden las relaciones humanas, con la organización del aula y con las formas de desarrollar la clase.

Por último, me inquietaba haber logrado en apenas tres semanas la articulación adecuada con la docente acompañante, pues habíamos realizado la planificación por correo electrónico.

\section{Empezar}

Desplazamos las mesas y las sillas de los lugares habituales, colocamos las sillas en círculo, como centro del círculo colocamos un pañuelo típico de color rojo y en cada silla dispusimos el material con el que trabajaríamos; y esperamos unos minutos a que las personas participantes se sentaran.

Inicié dando la bienvenida y explicando que íbamos a trabajar con un círculo de conversación, así que me referí, brevemente, a los principios, elementos estructurales, etapas del círculo y materiales de los que disponíamos; y pregunté si tenían consultas antes de iniciar.

Una de las participantes advirtió que en el centro del círculo hacía falta una pieza que representara a Ecuador; y seguidamente, un estudiante señaló que faltaba otra que representara a El Salvador. Así que aportaron una pulsera y una licencia de conducir, con lo cual se completaron las nacionalidades presentes y nos dispusimos a iniciar la experiencia. 
URL: http://www.una.ac.cr/educare

\section{La experiencia}

La ceremonia de apertura nos condujo a compartir las inquietudes, dificultades, experiencias, posibilidades y anhelos entorno al doctorado, al curso y a la investigación. Cada persona fue narrando lo que la canción decía de su estar ahí y en ese momento, hasta que la pieza completó la ronda.

-Respeto, solidaridad, confianza, constancia...- La canción de la ceremonia de apertura nos invitó a entrar en el círculo de conversación y también a los valores; pues cada participante fue diciendo, conceptualizando y escribiendo un valor, en una ficha; la cual fuimos pegando en una de las pizarras del aula.

Llegamos al consenso de los valores, después de una ronda con la pieza del diálogo. Las personas participantes consideraron que no había ningún valor más importante que otro y que aunque podían surgir nuevos en el futuro, en ese momento eran los que consideraban pertinentes al grupo y al contexto.

Si bien es cierto, las piezas del diálogo habían dado tres rondas al "círculo", llegamos a la hora del refrigerio sin coincidir con lo planificado. No obstante, esas primeras rondas permitieron a las personas verse, conocerse y relacionarse como compañeros y compañeras en una cultura compartida, y en donde enseñar y aprender fueron parte de un proceso esencial de incorporación en el mundo de la vida; al que refiere uno de los participantes de la siguiente manera:

Al principio, el temor a lo desconocido me hizo pasar por un periodo de introspección, reconociendo un espacio emocional, pero íntimo, en el que todo podía ser valioso y al mismo tiempo, debía seguir un patrón, socialmente establecido. Por ejemplo, el patrón establecido por las reglas de comportamiento establecidas para respetar la palabra de los demás y darnos el tiempo de escuchar.

Desde un punto de vista sociohistórico, en ese espacio microgenético (el círculo), podemos ser nosotros mismos, sin los formalismos de la racionalidad pura, basada en hechos, evidencias y datos objetivos. Esa objetividad solo llega a ser concebida como un punto de partida para el cuestionamiento libre, que busca nuevas opciones sobre lo conocido, pero que en la colectividad, se traduce en una asimilación-acomodación social que puede ser explicado desde un punto de vista piagetiano. El aprendizaje, según lo vivido, se da como un proceso libre pero intersubjetivo, surgido de la colectividad y sin posturas rígidas aristotélicas. (Caldwell, 2011, p. 1)

Otra de las participantes manifestó:

El trabajar con un grupo de compañeros de diferentes países, con diferentes costumbres, me hizo entender el significado de "un encuentro de nosotros con los otros". 
El tolerar, el respeto, la negociación, que fácil colgar los valores en un pizarrón, que difícil descubrir que nos hace falta mucho para tenerlos en su totalidad.

Este encuentro con los otros me ha conducido a iniciar de cero, mi perspectiva de la enseñanza cambió, la participación, el diálogo, la vergüenza se va acabando todos somos novatos al mismo tiempo, la mayoría nos respetamos y es lo que cuenta.

Para mí un modelo que se podría copiar: todos podemos hablar, hemos aprendido a estar juntos, hemos desarrollado habilidades que creíamos que no teníamos, hay espontaneidad, crítica, risas, lágrimas y nadie se cohíbe.

Un encuentro de nosotros con los otros del cual hemos aprendido a enseñar, con el cual otros aprenderían a romper el miedo por lo desconocido,

Quizá con este modelo, no únicamente un niño con síndrome de Down, si no miles de niños, adolescentes, jóvenes, adultos y adultos mayores que son excluidos continuamente de actividades comunes y a los cuales hemos etiquetado o clasificado, tengan la oportunidad de este encuentro con los otros, de descubrir un mundo de cosas nuevas. (Cavalini, 2011, p. 1)

Como manifiestan los estudiantes², esas primeras rondas con las piezas del diálogo permitieron cumplir con las dos primeras fases de la etapa de realización: Crear confianza y construir relaciones para el aprendizaje como un acto de libertad.

Así que, después de una pausa para compartir el refrigerio, volvimos al círculo y retomamos el diálogo con las preguntas propuestas en la etapa de preparación de la planificación didáctica.

Aquellas voces con "verdades" casi perfectas, casi incuestionables, absolutas y casi vacías, en la disposición del silencio, de la escucha y de la conversación, fueron movilizando a las personas participantes a narrarse en primera persona, a contarse en la experiencia, a significar desde el conocimiento propio, a poner en relación significados, a dar y buscar sentidos en lo vivido; como se refiere uno de los estudiantes de la siguiente manera:

En estas sesiones, el proceso que lleva al entendimiento paulatino pero desde el plano de lo subjetivo (reconociendo el valor de la diversidad de los sujetos que intervienen en el círculo), facilita la construcción teórica racional permeada por la inteligencia emocional colectiva.

(...) Es aquí donde surge lo inesperado: el sujeto individual, poco a poco deja de ser individuo y se convierte en sujeto social. Pero no deja de "ser" y se convierte en "un ser parte de", en donde la subjetividad de su punto de vista es valorada, apreciada y también parte de la asimilación-acomodación colectiva.

\footnotetext{
Los estudiantes han autorizado a la autora para que aparezcan los fragmentos citados de los diarios.
} 
URL: http://www.una.ac.cr/educare

(...) Partir de los sentimientos, compartirlos y reconocerlos, como un elemento válido en la construcción de conceptos o elaboraciones conceptuales a nivel colectivo, es un abordaje epistemológico totalmente nuevo para mí. Incluso lo es, la comunicación no necesariamente bidireccional. Es decir, en el círculo de conversación, muchas veces una persona comenta algo y si hay alguna discrepancia en el grupo, la réplica no necesariamente viene de inmediato. Hay que esperar el turno. Esperar mi turno, en ciertos momentos, no fue fácil. Pero curiosamente, al llegar mi participación, lejos de perder sentido de oportunidad, gané criterio. (Caldwell, 2011, pp. 1-2)

Llegamos a la hora de la ceremonia de cierre, sin haber abarcado todas las preguntas propuestas en la planificación, los tiempos de las voces habían circulado libremente siguiendo los ritmos de las piezas del diálogo, de la necesidad de profundizar y de quedarse en el tema que compartíamos y hacer del tema una experiencia. Al respecto una de las participantes manifiesta: "La sabiduría de la experiencia, nos enseña lo valioso de la vida" (León, 2011, p. 1)

Finalmente, la ceremonia de cierre nos trasladó por un momento a otra narración, a otro tiempo, a otro espacio, a otra forma de pensar y vivir la experiencia educativa; a otra forma que quizás no era tan distante, de lo que habíamos vivido. Nos preparó para salir del ambiente que habíamos construido y nos abrió a la posibilidad de pensar los temas abordados en contextos más próximos, más nuestros, y a pensar en esos contextos como ambientes susceptibles de transformación mediante acciones comunes.

\section{Seguimiento}

La etapa de seguimiento se realizó mediante el diario de aprendizaje redactado por las personas participantes y del que se extrajeron las apreciaciones manifestadas en el apartado anterior ${ }^{3}$.

\section{Consideraciones finales}

De la fundamentación, planificación, aplicación y experiencia del círculo de conversación como estrategia didáctica en el curso "PF-4237 Teoría de la Educación: Multiculturalidad y Educación", pueden destacarse las siguientes consideraciones:

- $\quad$ El "valor" de los valores. Es decir, de la riqueza de la construcción colectiva de normas sencillas de convivencia, que permiten a cada participante identificarse con el grupo y sentirse respetada.

\footnotetext{
La autora cuenta con la autorización de los estudiantes para citar los fragmentos del diario de aprendizaje.
} 
- La capacidad de cada persona participante de reconocerse en el "otro". Es decir, de interpretarse, conocerse y comprenderse como sujeto en una multiplicidad de historias y experiencias vividas por los "otros".

- La actitud de la escucha respetuosa y recíproca de cada persona participante.

- La posibilidad de cada sujeto de deconstruir la percepción individual y objetivada de un concepto específico y de reconstuirla inserta en un contexto complejo de dimensiones subjetivas y colectivas. Es decir, de dar significado y sentido a las palabras en el mundo en el que habitan.

- La confianza como un resultado que nace de la dinámica de dar legitimidad a lo vivido, como parte de un proceso de dar, recibir y agradecer y que pone en circulación conocimientos científicos y relacionales, que posibilitan nuevas acciones y nuevas realidades conectadas.

- La capacidad de las personas participantes de pensarse a sí mismas en lo que están haciendo y viviendo.

- La capacidad de cada una de las personas de abrirse a la crítica y a la creatividad como parte de un proceso formativo.

- La capacidad de cada una de las personas participantes de aproximar, tejer, combinar e imaginar un orden de relaciones con el contexto y en él, que deviene en una inteligencia política colectiva.

Si bien es cierto, la aplicación del círculo de conversación como estrategia didáctica abre la experiencia a la posibilidad de disfrutar de la acción educativa, de socializar el conocimiento y el saber, de reconocer la diferencia y la singularidad humana como una oportunidad de aprendizaje y convivencia, y de fomentar la formación como una acción continua de comunicación y relación humana, es pertinente también reconocer las dificultades y limitaciones de su aplicación frente a las normativas y prescripciones institucionales, en relación con el tiempo y espacio en el que debe suscitarse la acción formativa, y en cuanto a la forma en el abordaje de los contenidos del programa del curso. Ya que en esta experiencia no logramos abordar en una sesión de trabajo la totalidad de contenidos que proponía el programa, se hizo necesario realizar las sesiones siguientes con el uso de otras estrategias didácticas.

Las dificultades y limitaciones arriba planteadas me conducen a las palabras de María Zambrano cuando manifiesta que:

Trascender es eso ante todo: mediar, ir y venir entre lugares extremos, y si no es eso lo propio de lo humano, se quedará el hombre -y la mujer ${ }^{4}$ reducido a ser una criatura no muy diferente de las demás, se quedará privado de su situación singular en el mundo. (Zambrano, 2002, p. 107)

$4 \quad$ Lo que está entre guiones es de la autora. 
URL: http://www.una.ac.cr/educare

Palabras y experiencia que me invitan, nuevamente, a preguntarme: ¿A quién? ¿Cuándo? ¿Por qué? ¿Cómo? y ¿Para qué enseño? Preguntas que son parte de ese proceso de planificación, desarrollo y evaluación didáctica; que comprenden ese trayecto incesante de ida y vuelta entre lo pedagógico, lo curricular y lo didáctico, y entre lo epistemológico, metodológico y axiológico; que me recuerdan que la educación es una responsabilidad con el mundo y desde él, por la que no debo, como docente, dejar de interrogarme y de buscar nuevas formas de hacer.

\section{Referencias}

Arbelo, R. (2009). Llegaremos a tiempo. En álbum A las buenas y a las malas. [CD]. Los Angeles: Warner music.

Bernal, F. y Castillo, S. (2006). Justicia restaurativa en Costa Rica.: Acercamientos teóricos y prácticos. San José, Costa Rica: CONAMAJ.

Caldwell, E. (2011). Diario de reflexión. La experiencia de ser sujeto social y vivir la intersubjetividad: El círculo de conversación como estrategia para el aprendizaje. Universidad de Costa Rica.

Cavalini, H. (2011). Diario de reflexión. Nosotros con los otros. Universidad de Costa Rica.

De Vita, A. (2009). La creación social. Relaciones y contextos para educar. Roma: Carocci.

Elias, N. (1990). La sociedad de los individuos. Barcelona: Península.

Gadamer, H.-G. (2004). Hermenéutica de la Modernidad. Conversaciones con Silvio Vietta. (Trad. L. Elizaincín-Arrarás). Madrid: Minima Trotta.

García, J. (2007). Leer en la cara y en el mundo. Madrid: Herder.

León, A. (2011). Diario de reflexión. Educación: Entendimiento social y trabajo de aula. Universidad de Costa Rica.

Martínez, J. (2008). El viaje. Manuscrito inédito.

Martínez, Z. (2010). Círculos de paz. Hacia una cultura del diálogo y la reflexión en el ambiente laboral. Manuscrito inédito.

Pranis, K., Stuart, B. y Wedge, M. (2003). Peacemaking Circles. From Crime to Community [Haciendo círculos de paz. Del crímen a la comunidad]. New York: Living Justice Press.

Zambrano, M. (2002). L'art de les mediacions (Textos pedagògics) [El arte de las mediaciones. Textos pedagógicos]. Barcelona: Universidad de Barcelona. 